\title{
Controversies in Breast Cancer Screening Strategies
}

Presented by Therese Bevers, MD; Kirsten Bibbins-Domingo, PhD, MD, MAS; and Kevin C. Oeffinger, MD; facilitated by Mary Lou Smith, JD, MBA

\begin{abstract}
A panel discussion on controversies in breast cancer screening held recently at the NCCN 21st Annual Conference included only a few controversies. Representatives of the U.S. Preventive Services Task Force, American Cancer Society, and NCCN disagreed primarily on 2 main areas: when to start screening (ages 40, 45, or 50 years) and frequency of screening (annual vs biennial). Panelists from these organizations presented the rationales for their respective recommendations.
\end{abstract}

J Nat/ Compr Canc Netw 2016;14(5.5):651-653

At a panel discussion on controversies in breast cancer screening held recently at the NCCN 21st Annual Conference, representatives of the U.S. Preventive Services Task Force (USPSTF), American Cancer Society (ACS), and NCCN discussed the rationales for their organizations' respective recommendations on mammography.

First, explaining the grading system for USPSTF recommendations, Kirsten Bibbins-Domingo, $\mathrm{PhD}$, MD, MAS, from the University of California, San Francisco, and Chair of the USPSTF, said that grades $\mathrm{A}, \mathrm{B}$, and $\mathrm{C}$ all signify recommendations in favor of screening. However, these grades differ in certainty of evidence and magnitude of potential benefit, with A having the highest certainty for the largest net benefit. An I recommendation means that there is not enough evidence and represents a call for more research rather than a recommendation against screening, she said.

\footnotetext{
Presented by Therese Bevers, MD, The University of Texas MD Anderson Cancer Center, Houston, Texas, and Chair of the NCCN Guidelines for Breast Cancer Screening and Diagnosis; Kirsten Bibbins-Domingo, $\mathrm{PhD}, \mathrm{MD}, \mathrm{MAS}$, University of California, San Francisco, San Francisco, California, and Chair of the U.S. Preventive Services Task Force; and Kevin C. Oeffinger, MD, Memorial Sloan Kettering Cancer Center, New York, New York, and Chair of the American Cancer Society Breast Cancer Screening Guideline Panel; facilitated by Mary Lou Smith, JD, MBA, Research Advocacy Network, Naperville, Illinois.

Drs. Bevers, Bibbins-Domingo, and Oeffinger have disclosed that they have no financial interests, arrangements, affiliations, or commercial interests with the manufacturers of any products discussed in this article or their competitors. Ms. Smith has disclosed that she has served as a scientific advisor for Genentech, Inc.

Correspondence: JNCCN, 275 Commerce Drive, Suite 300, Fort Washington, PA 19034. E-mail: JNCCN@NCCN.org
}

The USPSTF assigns a grade $\mathrm{B}$ recommendation (moderate certainty of moderate net benefit) for biennial mammography screening for women aged 50 to 74 years at average risk. ${ }^{1}$ For women before age 50 years, the USPSTF recommendations state that screening is an individual decision, and women who place a higher value on potential benefit than potential harm may begin screening between ages 40 and 49 years. This is a $\mathrm{C}$ recommendation, suggesting that although there is moderate certainty of a net benefit, the magnitude of that benefit is small. Screening for women aged 75 years and older is an I recommendation, which means more research is needed.

"In the absence of evidence, clinicians and patients must use clinical judgment to determine the age to stop screening," Dr. Bibbins-Domingo said.

Discussing the ACS recommendations, Kevin C. Oeffinger, MD, from Memorial Sloan Kettering Cancer Center and Chair of the ACS Breast Cancer Screening Guideline Panel, explained that the ACS uses the "Grading of Recommendations Assessment, Development, and Evaluation" (GRADE) system, and recommendations are categorized as "strong" or "qualified." The only strong ACS recommendation is that women at average risk undergo regular mammography screening starting at age 45 years. Qualified ACS recommendations include that women ages 45 to 54 years should undergo screening annually; women aged 55 years and older should transition to biennial screening or contin- 
Bevers et al

ue to be screened annually; and women should have the opportunity to begin annual screening between the ages of 40 and 44 years. ${ }^{2}$ Two other qualified recommendations are that women should continue screening as long as their overall health is good and they have a life expectancy of 10 years or longer and that clinical breast examination is not recommended for women of any age at average risk.

Therese Bevers, MD, from The University of Texas MD Anderson Cancer Center, then discussed recommendations from the NCCN Clinical Practice Guidelines in Oncology (NCCN Guidelines) for Breast Cancer Screening and Diagnosis. She explained that the only category 1 recommendation in the guideline (which signifies "high-level evidence and uniform NCCN consensus that the intervention is appropriate") is to begin annual screening mammography for women at average risk at age 40 years after counseling for risks and benefits. ${ }^{3}$ The NCCN Guidelines recommend consideration of severe comorbid conditions limiting life expectancy and whether therapeutic interventions are planned. These guidelines do not establish an upper age limit for screening.

The different screening guidelines and recommendations generate a lot of confusion among clinicians and women, Dr. Bevers told listeners. A Breast Cancer Screening Consensus Conference was held in January 2016, to start the process of formulating a consensus statement. The combined group hopes to publish a manuscript that 8 organizations endorse. These organizations are USPSTF, ACS, NCCN, American College of Radiology, American Academy of Family Physicians, American College of Physicians, American College of Surgeons, and American College of Obstetricians and Gynecologists. Stakeholders from more than 22 other women's organizations and patient advocates also attended.

"We hope this will help clinicians understand where we agree, where we differ, and how we came to differ. Also, we are considering the recommendation to develop decision-making tools for women to use to determine which screening strategy is most appropriate for her," Dr. Bevers said.

The panelists agreed that there is no question that mammography is a highly effective method for reducing morbidity and mortality. Dr. BibbinsDomingo explained that the USPSTF formulated their recommendations based on 8 randomized controlled trials and more than 200 observational trials.

"The strongest evidence for decreasing the incidence of advanced cancer is in women age 50 and older. The evidence is mixed for women in their 40s," Dr. Bibbins-Domingo explained.

Dr. Oeffinger noted distinctions in the way the different groups consider mortality reduction from the randomized controlled trials (RCTs) and observation studies. Of note, the 8 randomized trials, designed to determine the efficacy of mammography, were largely conducted in the 1970s and 1980s using single view scans and older techniques.

"The duration of follow up is key. In general, the longer the follow up, the greater the mortality reduction. The systematic evidence review, conducted by Duke University's Evidence Synthesis Group and that provided the foundation of the ACS guidelines, used a 20\% mortality reduction based on meta-analyses of the randomized trials, while USPSTF assumes an $18 \%$ reduction," he noted. "However, if you look at contemporary, high-quality, real-world observational studies and properly adjust for confounders, the mortality reduction is closer to $35 \%$ in certain populations." he added.

According to Dr. Bevers, NCCN considered both the RCTs and observational studies, and found a significant mortality reduction with mammography screening.

"Women who are screened are less likely to be diagnosed at a more advanced stage and may not require aggressive therapy. Women aged 40 to 49 years had the highest number of years of life gained from screening, even though the reduction in mortality is greater in women in their 50s," she explained.

\section{Risks of Mammography Screening}

The panelists also discussed the risks of mammographic screening. For example, mammography carries a possibility of a false-positive test, necessitating further workup and possible biopsy, and creating anxiety. Approximately 50\% of women in their 40 s will have false-positive results; however, as women age, the rate of false-positive results decreases. However, because no prior mammograms are available for comparison to reassure the radiologist of a benign finding, false-positives occur more frequently with the first screening mammogram. This probably ac- 
counts for a number of the false-positive results seen in women in their 40s. If the starting age for mammographic screening is shifted to age 50 years, the false-positive rate at that age would increase.

"Overdiagnosis leading to overtreatment is a more rare and serious harm," said Dr. BibbinsDomingo. "The numbers are challenging and we can't measure this directly," she added.

"I agree that there is a level of overdiagnosis," Dr. Bevers concurred, "but it is challenging to quantitate it, and we don't know which women are overdiagnosed. It is important to understand the concept of overdiagnosis, but it is not a factor in the decision of when to start screening and the interval between screenings. This is a harm of screening that is incurred regardless of the age screening starts and whatever the interval is, as the mammographic finding-be it a calcification or other finding-will not go away unless it is treated," she noted. "To address the issue of overdiagnosis, we need to know which lesions won't progress," she added.

"The ACS considered overdiagnosis in developing the current guidelines," Dr. Oeffinger commented. Overdiagnosis "has been estimated at anywhere between $5 \%$ and $50 \%$, but we consider that it is likely less than $5 \%$," he said.

"When considering the harms of screening, we should be comparing them to the harms of not screening, not to 'no harms.' Women who are not screened are more likely to be diagnosed at a more advanced stage, require more intensive treatment, and are more likely to die from their disease," Dr. Bevers said.

\section{Screening Biennially Versus Annually}

The panel noted that the more often screening takes place, the higher the likelihood of both benefits and harms. The USPSTF position is that the most benefits accrue with biennial screening. Although annual screening provides some additional benefits, there are also more harms, Dr. Bibbins-Domingo explained.
"None of the studies tell us the optimal interval. We arrived at intervals by simulation modeling based on the data from the primary studies," she noted.

"The Breast Cancer Surveillance Consortium is a goldmine of data," Dr. Oeffinger noted. "In past studies, annual and biennial intervals were defined with broad intervals, so the ACS asked the consortium to look at tighter intervals. In younger premenopausal women, there was a significant difference, with annual screening being superior to biennial screening in detecting earlier stage, smaller-size tumors and negative nodal status. By the time women were postmenopausal, that significant difference was lost," he concluded.

Dr. Oeffinger noted that ACS recommendations provide women the option to switch to biennial screening at age 55 years. According to modeling by the Cancer Intervention and Surveillance Modeling Network (CISNET), approximately $80 \%$ of the benefit is retained with that approach. "ACS thinks it is important for women to be able to continue annual screening based on their preferences and values," Dr. Oeffinger said.

"NCCN made a different decision [from ACS] based on the same data," Dr. Bevers said. "Our recommendation is largely influenced by the fact that fewer women die as a result of annual screening compared with biennial screening. While this incurs more false-positives, the panel strongly believes that this outweighs the harms of a recall and possible biopsy," Dr. Bevers concluded.

\section{References}

1. Siu AL, U.S. Preventive Services Task Force. Screening for Breast Cancer: U.S. Preventive Services Task Force Recommendation Statement. Ann Intern Med 2016;164:279-296.

2. Oeffinger KC, Fontham ET, Etzioni R, et al. Breast cancer screening for women at average risk. JAMA 2015;314:1599-1614.

3. Bevers TB, Helvie M, Bonaccio E, et al. NCCN Clinical Practice Guidelines in Oncology for Breast Cancer Screening, Version 1.2015. Accessed April 10, 2016. To view the most recent version of these guidelines, visit NCCN.org 